\title{
Communication
}

\section{Semantic-Linked Data Ontologies for Indoor Navigation System in Response to COVID-19}

\author{
Abdullah Alamri \\ College of Computer Science and Engineering, University of Jeddah, Jeddah 23890, Saudi Arabia; \\ amalamri@uj.edu.sa
}

check for updates

Citation: Alamri, A. Semantic-Linked Data Ontologies for Indoor Navigation System in Response to COVID-19. ISPRS Int. J. Geo-Inf. 2021, 10, 607 https: / / doi.org/ 10.3390/ ijgi10090607

Academic Editors: Dana Dannélls and Wolfgang Kainz

Received: 13 July 2021

Accepted: 12 September 2021

Published: 14 September 202

Publisher's Note: MDPI stays neutral with regard to jurisdictional claims in published maps and institutional affiliations.

Copyright: (c) 2021 by the authors. Licensee MDPI, Basel, Switzerland. This article is an open access article distributed under the terms and conditions of the Creative Commons Attribution (CC BY) license (https:/ creativecommons.org/licenses/by/ $4.0 /)$.

\begin{abstract}
Indoor navigation has become more important these days due to the current situation worldwide in the aftermath of the outbreak of the COVID-19 pandemic, posing an unparalleled threat amounting to a humanitarian crisis on a global scale. Indoor navigation employs a variety of technologies, including Wi-Fi, Bluetooth, and RFID. Support for these technologies requires accurate information and appropriate processing and modeling to help and direct users of the optimal route to desired destinations and to monitor crowd density in order to maintain social distancing. This research will present a semantic indoor ontology model for indoor navigation and the reduction of human density in indoor space to ensure social distancing and prevent transmission. The proposed system is based on semantic representations of the components of navigation paths which, in turn, enable reasoning functionality. Despite the system's complexity, the evaluation revealed that it functions well.
\end{abstract}

Keywords: semantic model; routing algorithm; indoor space ontology; spatial databases; indoor navigation

\section{Introduction}

People use GPS receivers on their smartphones to navigate while driving. However, human movement from one place to another is not limited to outdoor spaces; therefore, there is a need for indoor navigation technology, which GPS may not be able to provide. Indeed, approximately $90 \%$ of human activities take place in an indoor environment [1].

A number of technologies specializing in indoor navigation, including positional techniques, have been developed over the past decade, with many researchers still working in this field. The outcomes of the latest indoor localization techniques are promising and can provide information to a variety of context-aware and location-based resources [2-4]. Traditionally, airport, museum, and shopping mall visitor localization services and equipment location detection services serve as examples of such services.

With the improvements of current indoor positioning devices such as RFID, Bluetooth, and Wi-Fi [5-7], data structures in indoor spaces have become critical foundations for a variety of applications. The development of an indoor positioning solution requires the creation of maps based on indoor floor plans, successful positioning techniques, algorithms, and the establishment of suitable facilities inside buildings, or other areas of localization.

Indoor navigation has become more important these days since the outbreak of the Covid-19 pandemic, which has quickly become a humanitarian issue on an unprecedented global scale. The virus continues to spread throughout nations, placing huge pressure on health systems in the fight to save lives. Companies and researchers worldwide are looking for ways to solve the problems associated with this virus and to stem the increasing crisis $[8,9]$.

Technology cannot totally prevent the spread of the pandemic, but it can inform, alert, and encourage those on the ground and those who need to be aware of the situation to take measures that can help reduce the spread of infection. Indoor navigation systems can play a critical role in helping to curb this pandemic and future outbreaks. The indoor navigation 
system can inform and direct users of the optimal route to desired destinations and can monitor crowd density to ensure social distancing, see Figure 1.

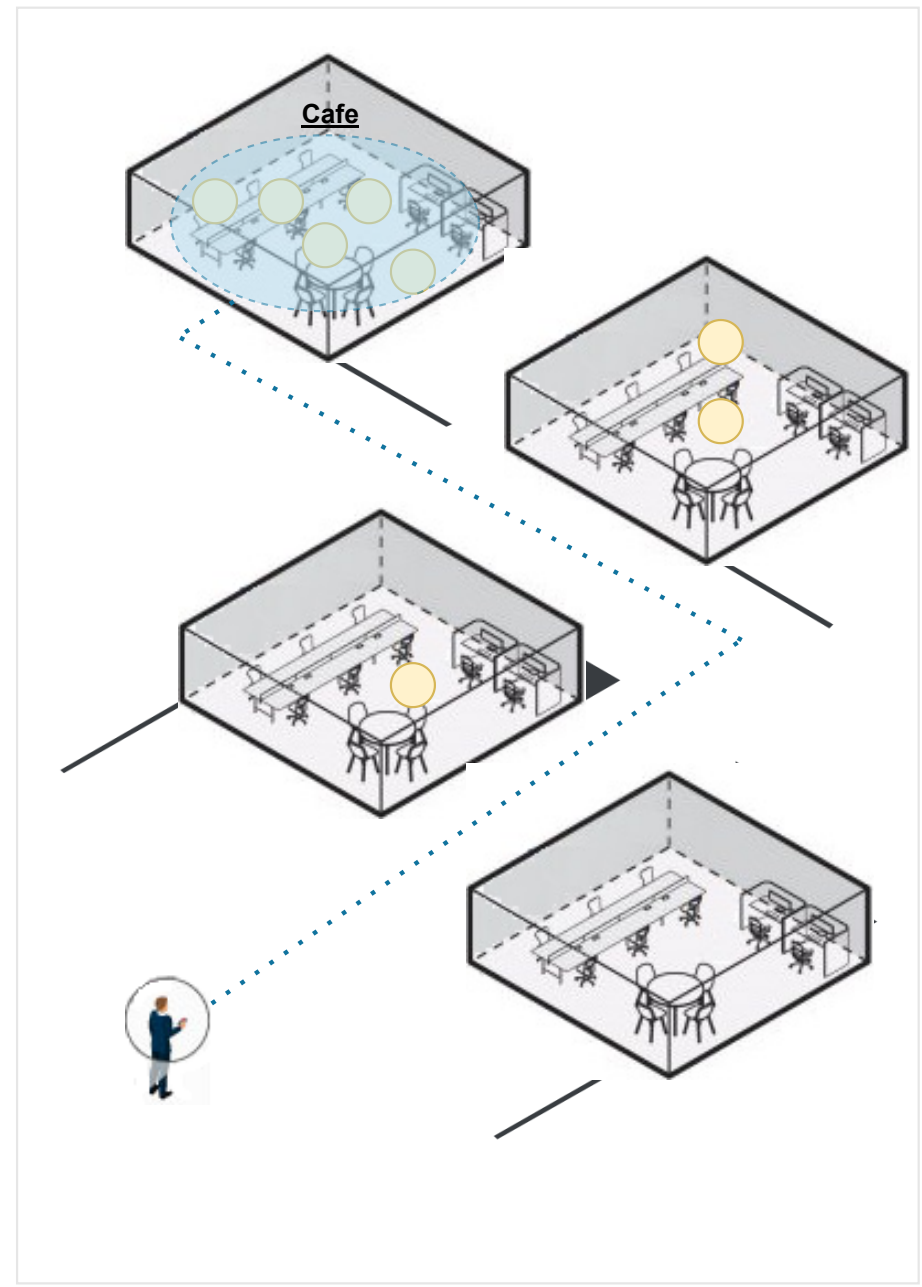

Figure 1. Monitoring crowd density to ensure social distancing and direct users using indoor navigation system.

In an indoor environment, the complexity of some buildings poses many challenges in terms of finding a route due to closed corridors, multiple floors, locked rooms, or other structural features. The captured indoor data requires proper processing and modeling. The design of ontology is fundamental for indoor searching, as it can increase search efficiency and provide the position of the object with greater accuracy. This research will present a semantic indoor ontology model to facilitate indoor navigation and reduce human density in indoor space to maintain social distancing and reduce the transmission of infection.

The proposed system is based on semantic representations of the components of navigation paths, which, in turn, enable reasoning functionality. Thus, we have established an indoor navigation ontology that fits both the route searching and the presentation tasks of the navigation system and monitoring of crowd density to maintain social distancing. This research paper addresses the following questions: 
- How to represent the complex indoor environment for pedestrians and conduct routing based on semantic representations?

- What are the functions needed to build a semantic indoor routing algorithm and monitor crowd density in indoor space?

The rest of the paper is structured as follows. Section 2 presents overview of semantic web modeling languages. Section 3 reviews the current state-of-the-art approaches of related works and studies that demonstrate the ontology that has been applied to the indoor navigation research. Section 4 presents the semantic indoor navigation architecture. Section 5 presents the experiment conducted to test the semantic-linked data ontologies for indoor navigation system in response to Covid-19. Section 6 concludes the paper and suggests recommendations for future study.

\section{Semantic Web}

The semantic web is a vision of the web of linked data in which data are given welldefined meaning, providing logical connections of terms. It is used in this study to design a semantic-linked data ontology for an indoor navigation system. The main elements of semantic web technology are [10-14] as follows:

- $\quad$ Resource Description Framework (RDF): a powerful triple-based representation language for Universal Resource Identifiers (URIs).

- $\quad$ RDF Schema (RDFS): a key Semantic Web technology providing data modeling and structured vocabularies for RDF data.

- Web Ontology Language (OWL): a type of knowledge representation languages which provides greater expressivity of objects and relations when describing domain knowledge.

- $\quad$ SPARQL: RDF query language for carrying out queries in data [15].

The semantic web's most important component is ontology. It organizes objects, data, as well as their attributes and relationships, within a domain of knowledge. In other words, the most popular definition of ontology is that it is data structure that efficiently describes a model of semantic representation and reasoning about structured knowledge of reality.

Definition 1. An ontology-structured is a 4-tuple $(C$, is_a, $P, \mathfrak{d})$, where $C$ is a set of concepts $\left(c_{1}, c_{2}, \ldots, c_{n} \mid c_{i} \in C\right)$, is_a is a partial order relation on $C, P$ is a set of property names and $\mathfrak{d}$ : $P\left(c_{i} \times c_{j}\right)$ describes how concepts are related.

In ontology, the term 'concept' refers to an abstract group, set, or series of objects that represent individuals (instances) in the discourse domain. A property can be further described as an "object property or data property", which expresses relationships between two concepts in each domain. A data property describes the relationship between an actual concept and the literal meaning, while object property describes the relationship between entities (instances) of two concepts $[14,16,17]$. Figure 2 shows the conceptual components of semantic DL ontology. 


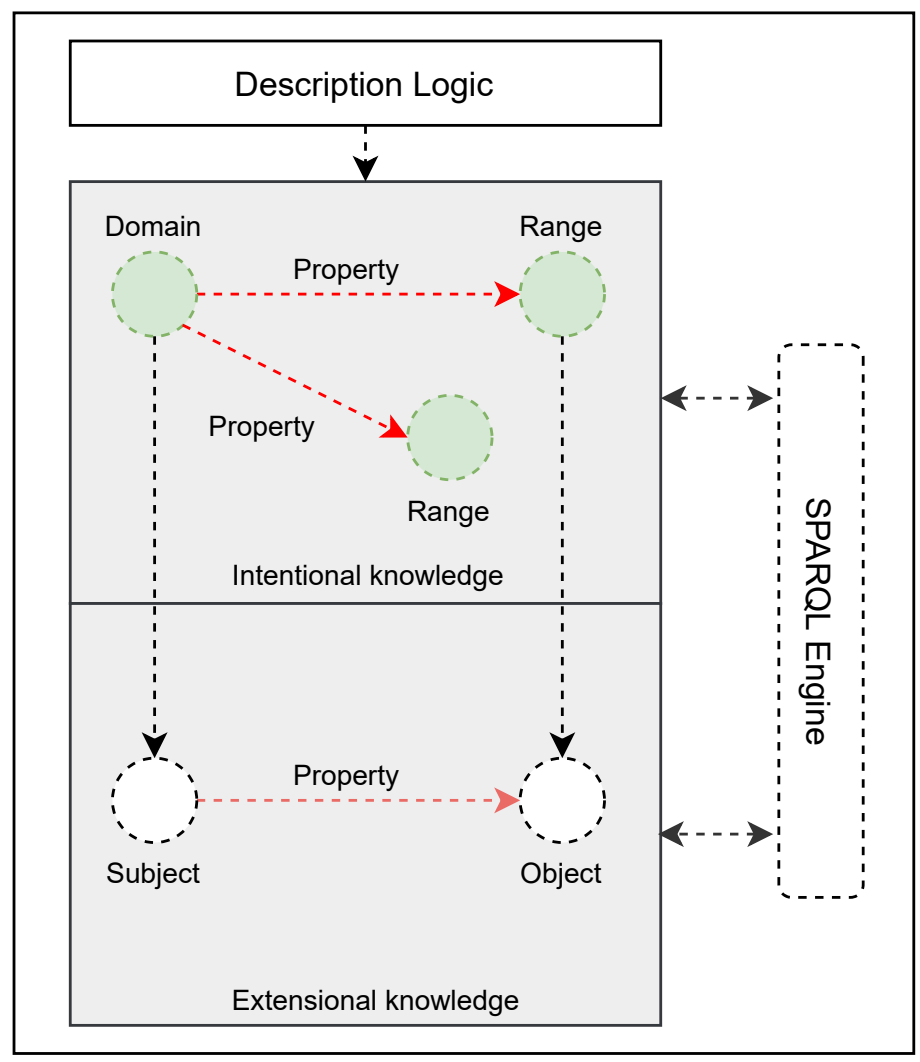

Figure 2. Semantic DL ontology knowledge.

\section{State-of-the-Art}

This section presents the review of related literature and studies that have applied ontology to indoor navigation research. The problem of organizing and sharing information in indoor navigation can be solved effectively using an ontology-based semantic model. The primary aim of using ontology for indoor navigation is to provide a semantic definition of particular events that occur within indoor environments.

Ontology has been applied to indoor navigation research such as IndoorGML [18]. IndoorGML provides a standard data model for modeling indoor spatial information. Dudas et al. [19] present ONALIN, an ontology and algorithm for indoor routing that takes into account the ADA (American Disability Act) requirements, among other items. Anagnostopoulos et al. [20] proposed a hybrid modeling technique named OntoNav which comprises semantic information for user navigation and an ontological framework for handling routing requests. Scholz and Schabus [21] developed an indoor navigation ontology to enable autonomous navigation in the indoor space for the movement of products in a manufacturing environment. Geodint [22] applied the traditional shortest path algorithm in a derived graph model for navigation. Wang et al. [23] proposed an ontology for representing a semantic location in indoor navigation models. Sriharee [24] developed an indoor navigation system based on OWL-defined symbolic knowledge. Lee et al. [25] proposed a location-based service that focuses on indoor activities at a university, using ontology-based semantic queries.

The main difference in our model compared to the mentioned related work is the use of semantic query languages, reasoning, and new ontology design and implementation for the computation of optimal routes. The traditional map navigation service primarily focuses on the route to a single destination, such as shortest path, which is more concerned with the given starting and ending points. However, in order to monitor crowd density to ensure social distancing and reduce the transmission of disease, there is the need to design and implement a new specialized ontology route algorithm for indoor spaces. 


\section{Semantic Indoor Navigation Architecture}

The semantic indoor navigation architecture is comprised of several building blocks as shown in Figure 3. The architecture has three main components: semantic navigation service, semantic schema path selection service, and density rules service. The proposed architecture's components are detailed as follows.

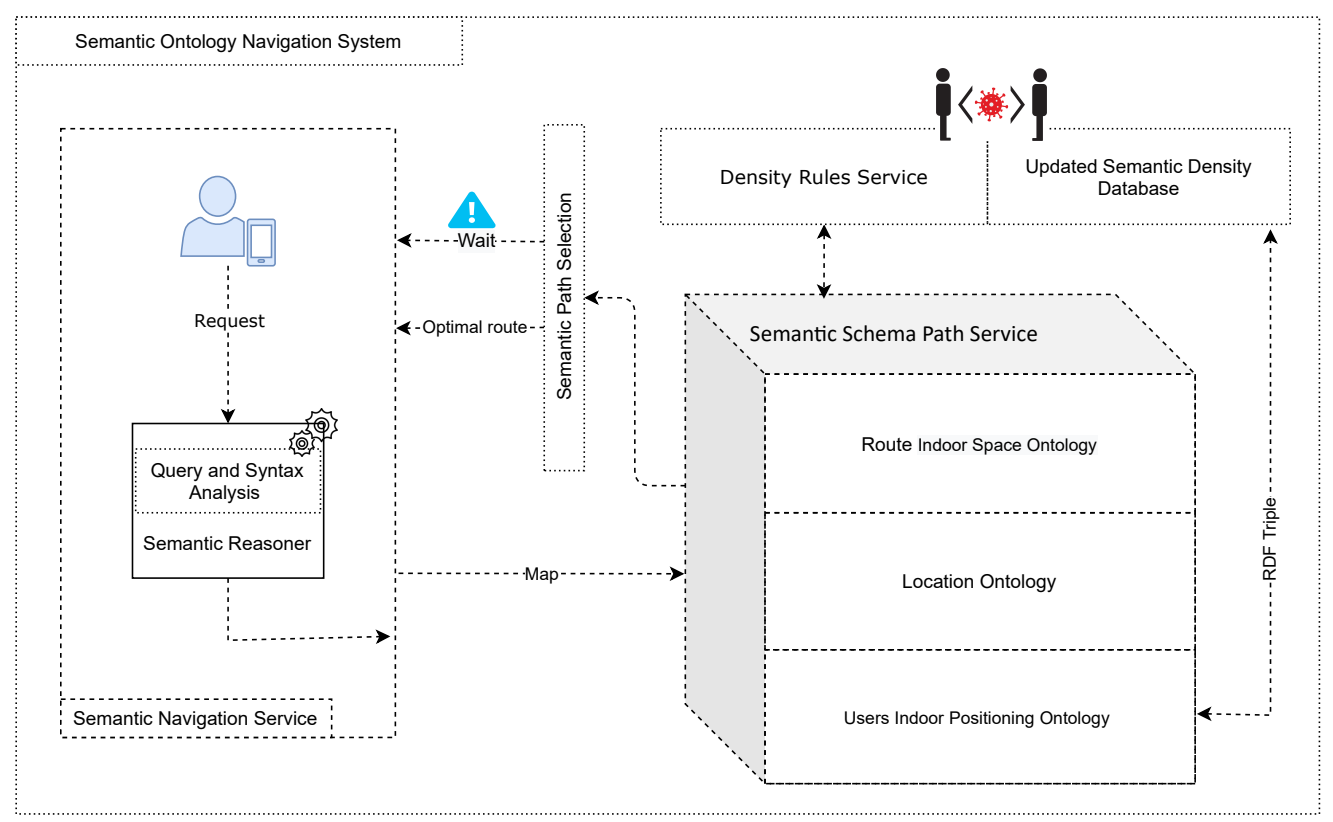

Figure 3. Semantic indoor navigation architecture.

\subsection{Semantic Navigation Service}

This service is the main interface between the user and the system. It receives a user request for navigation and responds with the optimal paths. Considering the usability of indoor location-based services, the semantic navigation service is applied in a two-step process. First, the user writes a query to get to a specific room in the building. The semantic reasoner receives an input query. The query is parsed to identify the destination room. Subsequently, the user's current position is also calculated and displayed on a mobile app.

Definition 2. Given destination room $\mathrm{v}$ and user-mobile-node $\mathrm{u}$ that presents the user location, navigation service forwards: $(u, v) \stackrel{\text { map }}{\rightarrow}$ SPS:Schema Path Service.

\subsection{Semantic Schema Path Selection Service}

This service presents the semantic indoor navigation ontology knowledge base, which provides the main functionality of our proposed system and is responsible for the selection of the best traversable navigation path for the user. In this service, there is a need to consider complex indoor space information such as multiple floors in the building, users' indoor position, and routes to find the optimal route for users inside a building. Figure 4 depicts the architecture of the semantic knowledge base for the indoor navigation system described in this section. It gives the definitions of ontologies and shows how they relate to each other to provide support for all facets of navigation. The framework architecture of the semantic knowledge base employs three types of domain-specific ontologies: route indoor space ontology, location ontology, and the user's indoor positioning ontology. 


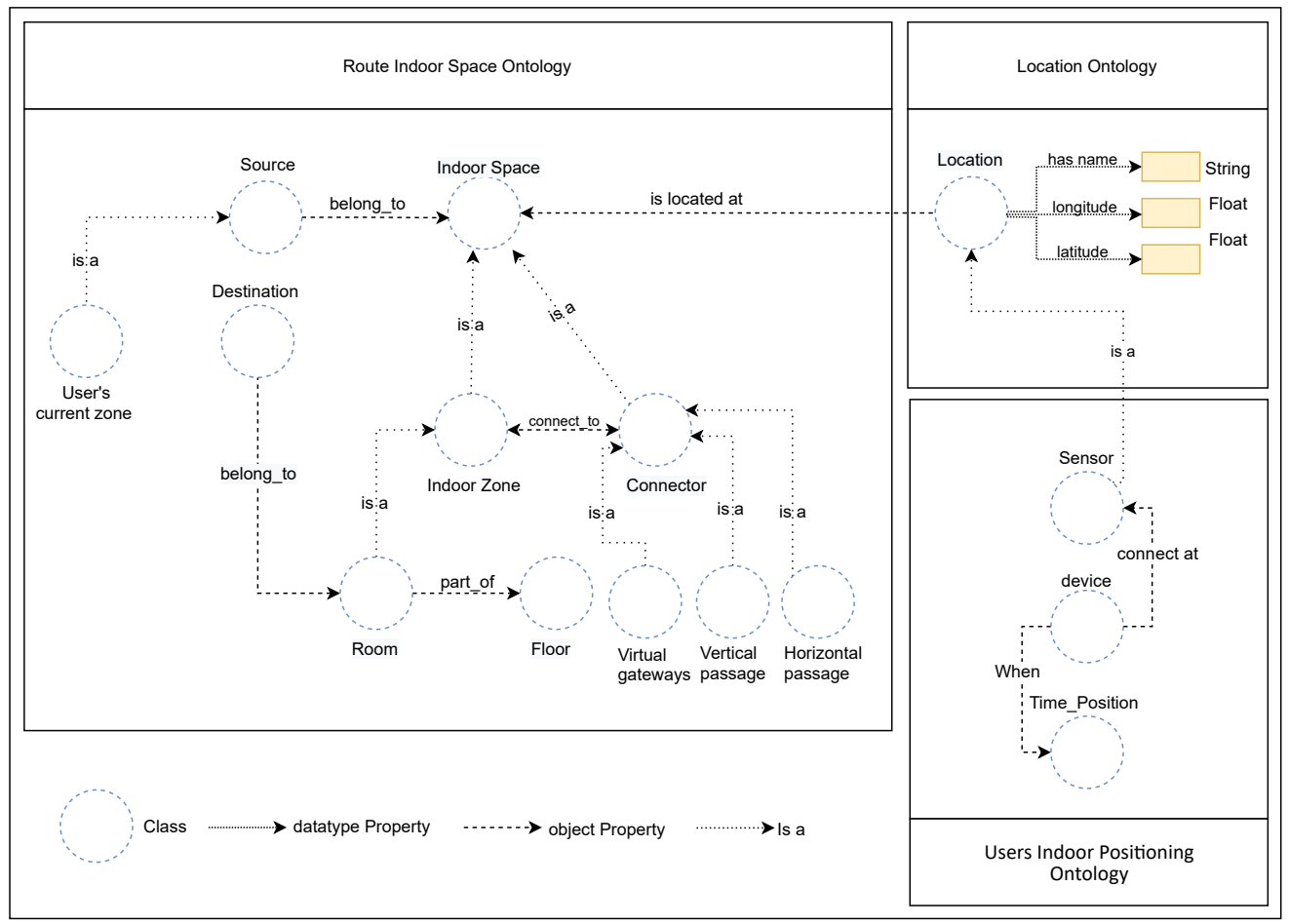

Figure 4. Semantic knowledge base for the indoor navigation system.

\subsubsection{Route Indoor Space Ontology}

As the indoor space becomes complex, the semantic partition of the data model for indoor space should efficiently support complex features of indoor space. Figure 5 shows the taxonomy of indoor space ontology. The taxonomy of indoor space ontology includes two main concepts: indoor zone and connector. The indoor zone defines the main subclass which comprises the room and the floor to which it belongs in order to demonstrate the part-whole hierarchy of floors in buildings. The connector class represents the connection between the indoor zone and a different floor of the building. The connector has three primary subclasses: horizontal passage, vertical passage, and virtual gateways.

- Horizontal passages are connecting corridors on the same floor such as doors that separate zones (i.e., rooms) with small physical extensions on the same floor.

- Vertical passages are connection between corridors on different floors. Elevators, escalators, and stairs are examples of vertical passages and are distinguished according to their characteristics (e.g., accessibility).

- Virtual gateways are joined corridors that are not separated by doors.

The route indoor space ontology is utilized to define the way a user navigates from a starting location to the destination.

Definition 3. The Route output in ontology will be applied using a route container to describe group of things, Route_container $R_{c i}=\left(u, c_{i 1} \ldots c_{i n}, l_{i}, v\right)$ where

- $\quad u$ is determined from the user's current zone location.

- $\quad c_{i 1} \ldots c_{\text {in }}$ represents the connection points that will be drawn from user to destination on the same floor.

- $\quad l_{i}$ represents the connection point to connect to other floor levels.

- $v$ represents the destination zone.

We illustrate the route container by an example which is shown in Figure 6. 


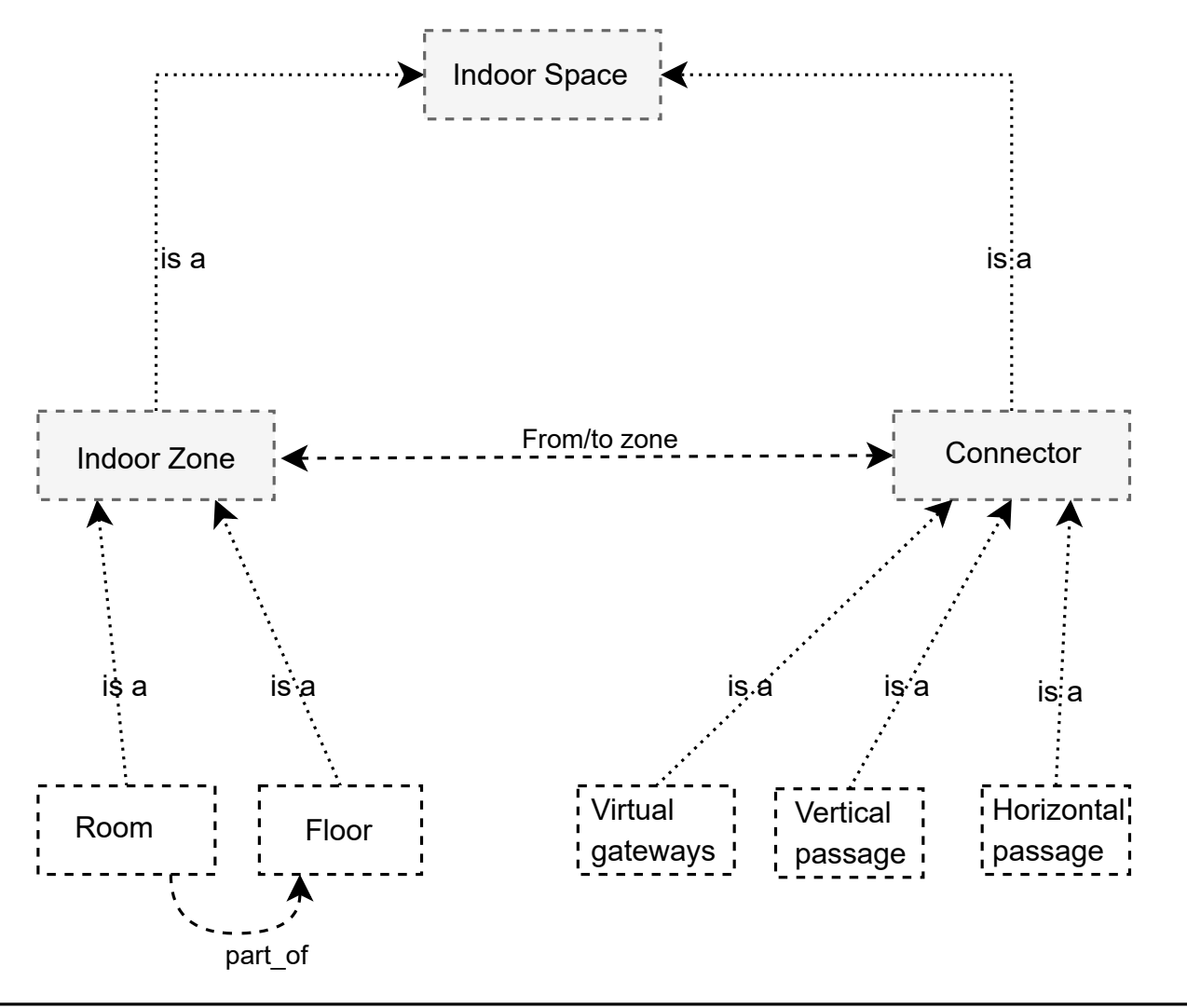

Figure 5. The taxonomy of indoor space ontology.

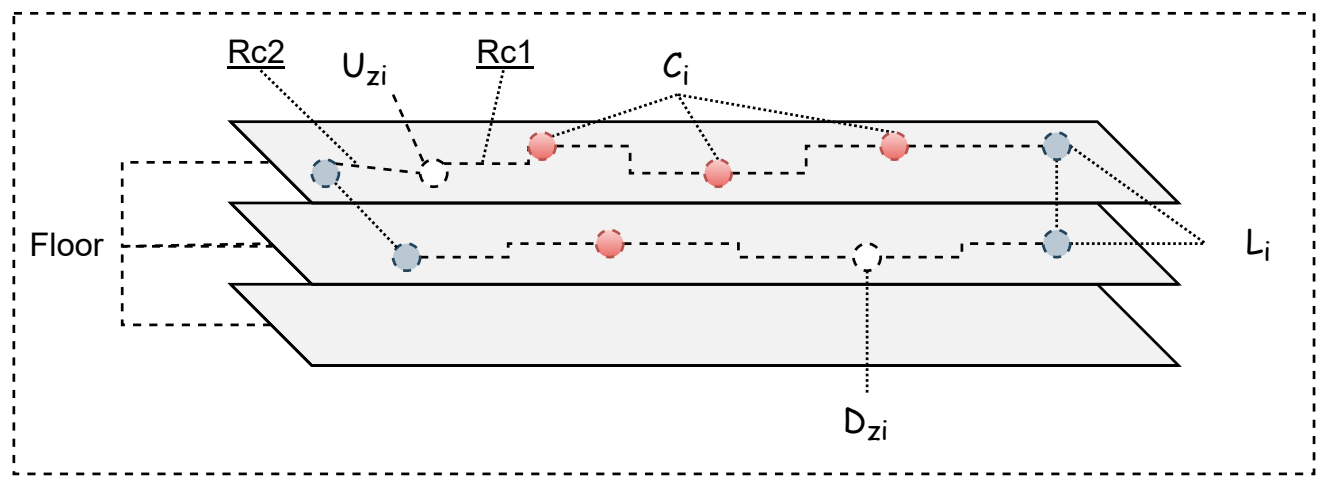

Figure 6. Route container.

\subsubsection{Location Ontology}

The location model shows location, direction, real-world topology, and moving objects (e.g., users carrying location-aware mobile devices). In the location ontology model, we can obtain rich location semantics of the indoor space and make the location information possible to understand. Location ontology is designed to identify the location of users in the indoor space.

\subsubsection{Users Indoor Positioning Ontology}

The last location and time where and when, respectively, the users were active are determined from the ontology. Sensors detecting Bluetooth and Wi-Fi units have been successfully used and applied for indoor positioning as they collect information and determine the current location of the user's mobile device. The ontology is designed using concepts: "time_position" and "location", and uses some of the existing ontology. In 
particular, OWL time ontology is used to express the time information such as temporal unit and temporal entities [26].

Definition 4 (Indoor Positioning). An indoor position of users, $I P_{u i}=p_{t i}$ represents sequence of triple points, $p_{t i}=\left(u_{i}, t_{i}, l_{i}\right)$, where $u_{i}$ is a user's mobile identifier; $t_{i}$ is the time that $p_{t i}$ was collected; and $l_{i}=\left(l_{o i}, l_{a i}, z_{i}\right)$ corresponds to the longitude, latitude, and zone, respectively, of the user at time $t_{i}$.

More precisely, smartphone sensors on the user's mobile can be linked to devices deployed nearby such as Wi-Fi or Bluetooth beacon to get the current user location measurements and compute the user positioning information by using device location and direct signal strength measured by the device signal receiver. The information on the users indoor positioning can be stored as a semantic triple store.

\subsection{Density Rules Service}

When there are many people in an enclosed indoor space, this can increase the threat of Covid-19 infection spreading from one person to another. The density rule service is applied to limit the number of users gathering in an indoor zone at the same time. The density limitation can be specified by manual entries on the basis of human decisions and indoor zone size. The density rule service limits users' access to specific indoor zones based on the updated semantic density database which is used to store information about the last location of the users or when they were last active.

In order to motivate our scientific work, in the following, we give an example of a scenario where a user wants to access a particular room. In this case, if the user wants to access indoor zone ' $\mathrm{R} 6$ ' in the building, and the density rule stipulates no more than 4 users per zone, the optimal route to access zone 'R6' will be selected based on route and zone density. If all routes and destination zones have a density of more than 4 , then the system sends the alert alarm "wait for 3 minutes and try again". Otherwise, optimal routes to zone 'R6' will be selected. Figure 7 illustrates the node and link concepts of the ontology. Semantic indoor navigation can be summarized as the pseudocode shown in Algorithm 1. Semantic indoor path selection is shown in Algorithm 2. Furthermore, the density rule service is shown in Algorithm 3.

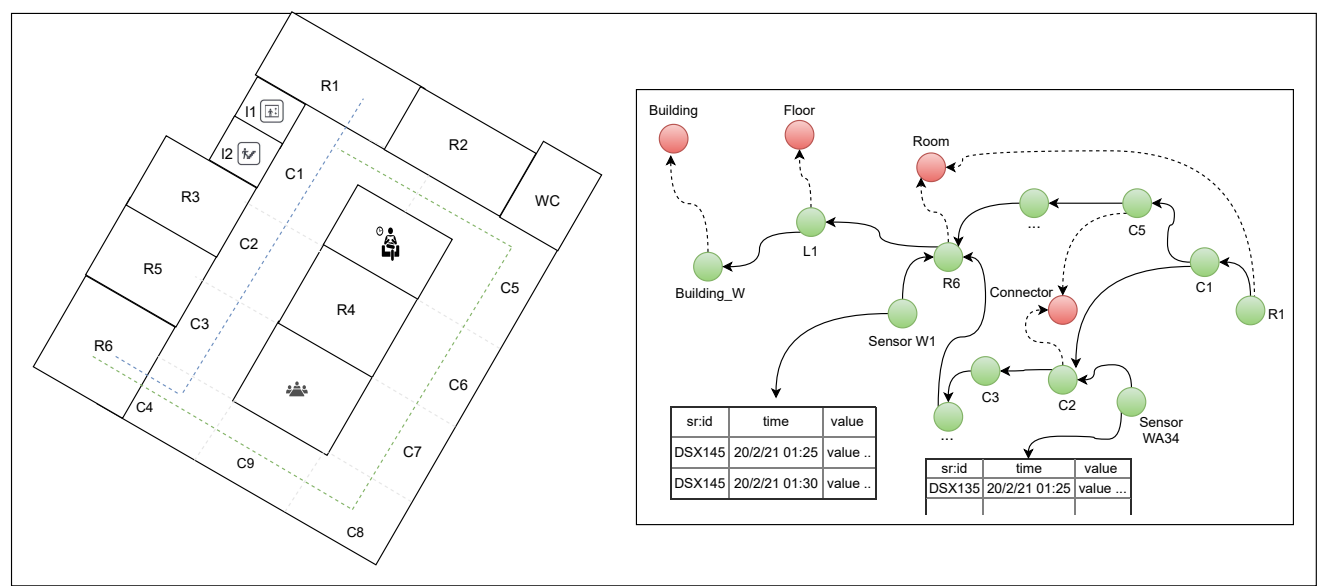

Figure 7. Example of nodes and links. Class nodes are red and instance nodes are green in color. 

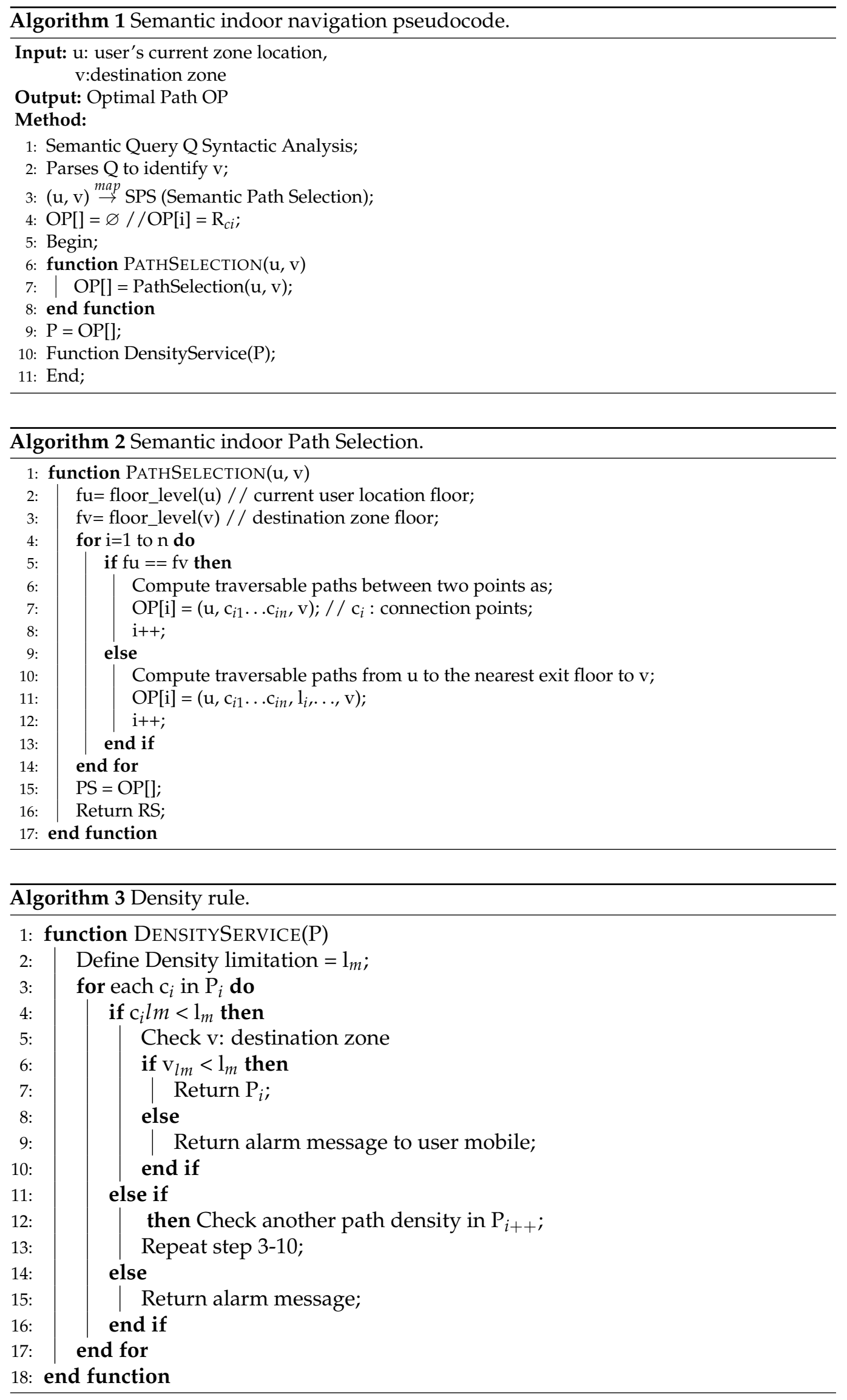


\section{Validation Experiments and Analysis}

The results of this testing and analysis process will reveal how well the system performs. There is one aspect that we will focus on in this experiment and that is the execution time. To assess the performance of the proposed semantic-linked data model for indoor navigation system, the experiment was run on a 64-bit Windows 10 Enterprise machine with an Intel Core i7 processor and 16 GB of RAM. The semantic indoor navigation system was implemented in Java and the Jena framework was used to handle the semantic ontology files. This paper does not cover the implementation of a mobile app.

In this experiment, we generated a virtual dataset for both the indoor space ontology which is a hypothetical two-floor building and generated semantic users' indoor ontology. We assume the indoor positioning node devices are stationary and their positions are known. We also assume the users' mobile nodes (moving object instances) that present positioning location of users' nodes are stored in semantic database.

The initial goal of our experimental effort has been to demonstrate the performance of the proposed semantic-linked data model for the indoor navigation system. We conducted several tests based on the following two parameters.

- Impact of number of moving object instances in building on system performance.

- The costs of the semantic routing on the same or different floors.

Results and Discussion: First, we investigate the impact of increased the moving object instances on system performance. Figure 8 shows the average response time and shows that when the number of moving objects increases, the response time of the semantic-linked data ontologies for indoor navigation system increases slightly. These results indicate the response time obtained by the semantic reasoner, the routing algorithm, and the density rules algorithm.

Furthermore, we measure the semantic routing costs for the proposed method, taking into account the number of nodes expanded (in the same or different floors). Figure 9 shows the response time of the semantic routing that evaluates the path search based on the distance between the reference and target objects. These experiments demonstrate the costs incurred by the semantic routing in terms of total execution time due to the number of nodes expanded.

Although the results reported in this article provide acceptable performance, we are aware that the experiment can be improved which will form the basis of our future research. We developed a semantic indoor navigation ontology that suits both the route searching and presentation tasks of the navigation system and crowd density monitoring to ensure social distancing. The proposed system is currently in its development phase, and we aim to evaluate its effectiveness and performance through its real-world deployment. Thus, future work on real-world experiments will be used to improve this framework for practical application. Furthermore, we aim to investigate positioning error and positioning accuracy of routing.

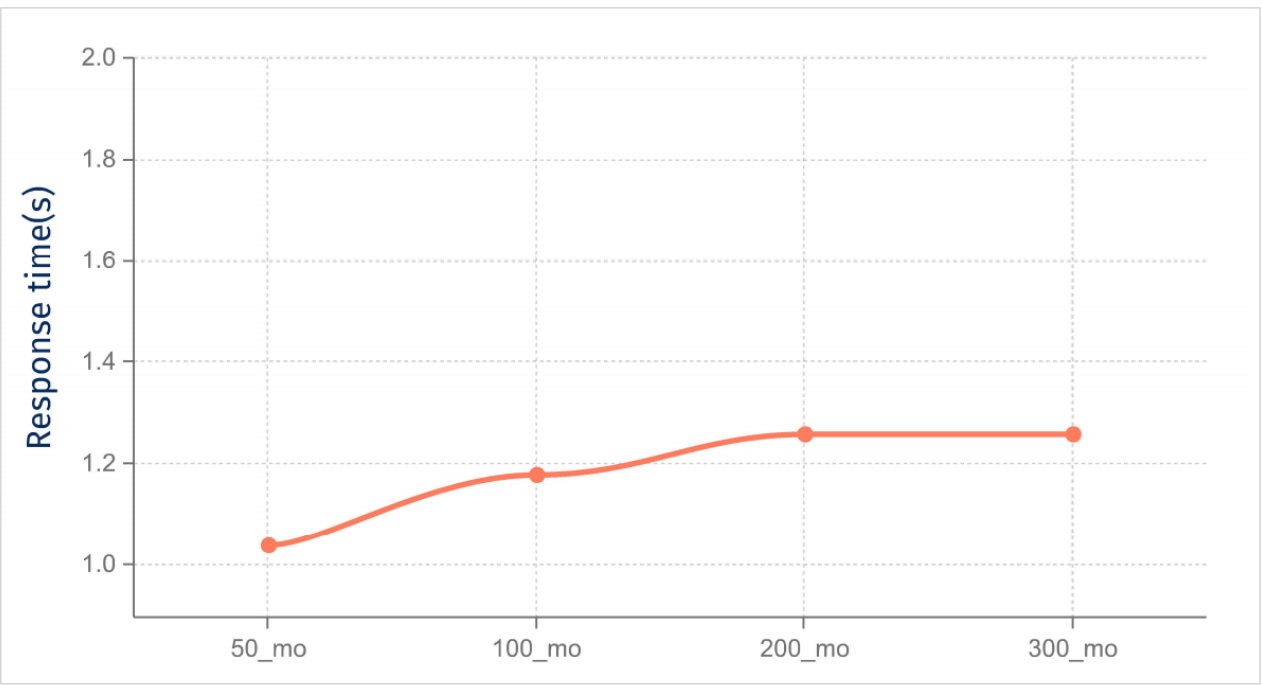

Figure 8. Impact of number of moving objects in building on system performance. 


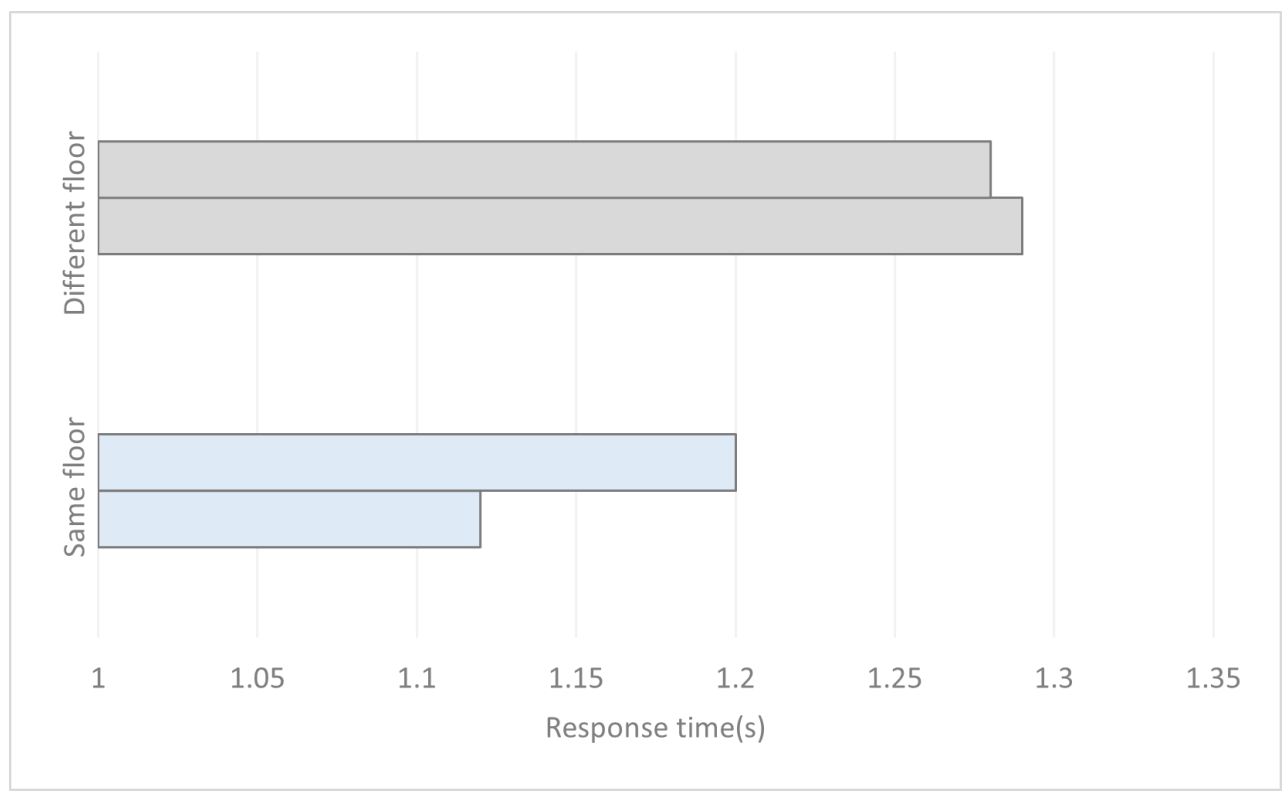

Figure 9. Performance of the semantic routing in four cases based on the distance between the reference and target objects.

\section{Conclusions}

In this study, we developed semantic-linked data ontologies for indoor navigation systems. The proposed system suits both the navigation system's route searching and presentation tasks, as well as providing crowd density monitoring to preserve social distance. It can play a critical role in helping to curb this pandemic and future outbreaks by informing, alerting, and encouraging those on the ground and those who need to be aware of the situation, thus reducing significantly the incidence of transmission. From a functional perspective, the semantic indoor navigation architecture consists of three main components: semantic navigation service for representing the interface between the user and the system, semantic schema path selection service for representing the complex indoor environment based on semantic representations, and density rules service for monitoring crowd density in indoor space. With the help of the proposed semantic-linked data ontologies for indoor navigation systems, users can find the place in a building easily without having to come into contact with anyone which would impede the spread of the Covid-19 virus.

Future work will improve the experiment detailed in this research and obtain results for larger data size. Furthermore, future work on actual experiments is intended to expand this framework for practical use. In addition, there are several challenges that need to be addressed. We intend to evaluate the impact on the proposed system of privacy protection and security issues.

Funding: This research received no external funding.

Data Availability Statement: The raw/processed data required to reproduce the above findings cannot be shared at this time as the data also forms part of an ongoing study.

Conflicts of Interest: The authors declare no conflict of interest.

\section{References}

1. Klepeis, N.E.; Nelson, W.C.; Ott, W.R.; Robinson, J.P.; Tsang, A.M.; Switzer, P.; Behar, J.V.; Hern, S.C.; Engelmann, W.H. The National Human Activity Pattern Survey (NHAPS): a resource for assessing exposure to environmental pollutants. J. Expo. Sci. Environ. Epidemiol. 2001, 11, 231-252. [CrossRef] [PubMed]

2. Dayekh, S.; Affes, S.; Kandil, N.; Nerguizian, C. Cooperative localization in mines using fingerprinting and neural networks. In Proceedings of the 2010 IEEE Wireless Communication and Networking Conference, Sydney, Australia, 18-21 April 2010 ; pp. 1-6.

3. Qiao, G.Z.; Zeng, J.C. Localization algorithm of beacon nodes chain deployment based on coal mine underground wireless sensor networks. J. China Coal Soc. 2010, 35, 1229-1233. 
4. De Blasio, G.; Quesada-Arencibia, A.; García, C.R.; Molina-Gil, J.M.; Caballero-Gil, C. Study on an indoor positioning system for harsh environments based on Wi-Fi and bluetooth low energy. Sensors 2017, 17, 1299. [CrossRef] [PubMed]

5. Zhu, L.; Yang, A.; Wu, D.; Liu, L. Survey of indoor positioning technologies and systems. In Life System Modeling and Simulation; Springer: Shanghai, China 2014; pp. 400-409.

6. Mautz, R. Indoor Positioning Technologies; ETH Library: Zürich, Switzerland, 2012.

7. Mendoza-Silva, G.M.; Torres-Sospedra, J.; Huerta, J. A meta-review of indoor positioning systems. Sensors 2019, $19,4507$. [CrossRef] [PubMed]

8. Alamri, A.; Alamri, S. Live Data Analytics with IoT Intelligence-Sensing System in Public Transportation for COVID-19 Pandemic. Intell. Autom. Soft Comput. 2021, 27, 441-452. [CrossRef]

9. Lee, B.; Lee, M.; Bibliowicz, J.; Goldstein, R.; Mogk, J.; Tessier, A. Simulation and Visualization of Virus Transmission for Architectural Design Analysis. In ACM SIGGRAPH 2021 Talks; Association for Computing Machinery: New York, NY, USA, 2021; SIGGRAPH '21. [CrossRef]

10. Alamri, A. Ontology middleware for integration of IoT healthcare information systems in EHR systems. Computers 2018, 7, 51. [CrossRef]

11. Quilitz, B.; Leser, U. Querying distributed RDF data sources with SPARQL. In Proceedings of the European Semantic Web Conference, Karlsruhe, Germany, 26-30 October 2008; Springer: Berlin/Heidelberg, Germay, 2008; pp. 524-538.

12. McGuinness, D.L.; Van Harmelen, F. OWL web ontology language overview. W3C Recomm. 2004, 10, 2004.

13. Klyne, G. Resource Description Framework (RDF): Concepts and Abstract Syntax. 2004. Available online: http://www.w3.org/ TR/2004/REC-rdf-concepts-20040210/ (accessed on 12 July 2021).

14. Sengupta, K.; Hitzler, P. Web ontology language (OWL). In Encyclopedia of Social Network Analysis and Mining; Springer: New York, NY, USA, 2014.

15. DuCharme, B. Learning SPARQL: Querying and Updating with SPARQL 1.1; O'Reilly Media, Inc.: Sevastopol, CA, USA, 2013.

16. Alamri, A. Semantic health mediation and access control manager for interoperability among healthcare systems. In Data Analytics in Medicine: Concepts, Methodologies, Tools, and Applications; IGI Global: Hershey, PA, USA, 2020; pp. 169-181.

17. Kalibatiene, D.; Vasilecas, O. Survey on ontology languages. In Proceedings of the International Conference on Business Informatics Research, Riga, Latvia, 6-8 October 2011; Springer: Berlin/Heidelberg, Germay, 2011; pp. 124-141.

18. Kim, J.S.; Yoo, S.J.; Li, K.J. Integrating IndoorGML and CityGML for indoor space. In Proceedings of the International Symposium on Web and Wireless Geographical Information Systems, Seoul, Korea, 29-30 May 2014; Springer: Berlin/Heidelberg, Germay, 2014; pp. 184-196.

19. Dudas, P.M.; Ghafourian, M.; Karimi, H.A. ONALIN: Ontology and algorithm for indoor routing. In Proceedings of the 2009 Tenth International Conference on Mobile Data Management: Systems, Services and Middleware, Taipei, Taiwan, 18-20 May 2009; pp. 720-725.

20. Anagnostopoulos, C.; Tsetsos, V.; Kikiras, P. OntoNav: A semantic indoor navigation system. In Proceedings of the 1st Workshop on Semantics in Mobile Environments (SME05), Ayia, Citeseer, 9 May 2005.

21. Scholz, J.; Schabus, S. An indoor navigation ontology for production assets in a production environment. In Proceedings of the International Conference on Geographic Information Science, Vienna, Austria, 24-26 September 2014; Springer: Berlin/Heidelberg, Germay, 2014; pp. 204-220.

22. Matuszka, T.; Gombos, G.; Kiss, A. A new approach for indoor navigation using semantic webtechnologies and augmented reality. In Proceedings of the International Conference on Virtual, Augmented and Mixed Reality, Las Vegas, NV, USA, 21-26 July 2013; Springer: Berlin/Heidelberg, Germay, 2013; pp. 202-210.

23. Wang, X.; Shang, J.; Yu, F.; Yan, J. Indoor semantic location models for location-based services. Int. J. Smart Home 2013, 7, 127-136.

24. Sriharee, G. A symbolic-based indoor navigation system with direction-based navigation instruction. Procedia Comput. Sci. 2015, 52, 647-653. [CrossRef]

25. Lee, K.; Lee, J.; Kwan, M.P. Location-based service using ontology-based semantic queries: A study with a focus on indoor activities in a university context. Comput. Environ. Urban Syst. 2017, 62, 41-52. [CrossRef]

26. Hobbs, J.R.; Pan, F. Time ontology in OWL. W3C Work. Draft 2006, 27, 133. 